\title{
$\frac{a b]}{a b}$ \\ Journal of Applied Biosciences 67:5261 - 5270 \\ Procédés de production d'aliments non conventionnels pour porcs à base de déchets de mangues et détermination de leurs valeurs alimentaires au Burkina Faso
}

\author{
KIENDREBEOGOTimbilfou ${ }^{1 *}$, MOPATE LOGTENE Youssouf ${ }^{2}$, IDO Georges ${ }^{3}$ et KABORE-ZOUNGRANA \\ Chantal-Yvette 4 \\ ${ }^{1}$ Institut de l'Environnement et de recherches Agricoles (INERA), Département Productions Animales (PA), Station de \\ Farakobâ, 01 BP 910 Bobo-Dioulasso (Burkina Faso) 01; Tél. + 22670223003 / +226 20962946 ; E-mail: \\ timbilfou@gmail.com \\ 2Laboratoire de Recherches Vétérinaires et Zootechniques (LRVZ), Farcha, B.P. 433, N'Djaména, Tchad. Chercheur \\ associé au LERNSE de l'Université Polytechnique de Bobo-Dioulasso \\ 3Unité de séchage TENSYA/GUAMPRI de Toussiana, région des Haut-Bassins, Burkina Faso \\ 4Laboratoire d'Etudes et de recherches sur les Ressources Naturelles et les Sciences de l'Environnement (LERNSE), \\ Université polytechnique de Bobo-Dioulasso (UPB), 01B.P.1091 Bobo-Dioulasso 01 Burkina Faso \\ *Auteur pour la correspondance : timbilfou@gmail.com
}

Original submitted in on 22 ${ }^{\text {nd }}$ April 2013 Published online at www.m.elewa.org on 30 th July 2013.

https://dx.doi.org/10.4314/jab.v67i0.95047

\section{RÉSUMÉ}

Objectif : L'objet de l'étude a été de mettre au point un procédé de production d'aliments non conventionnels à base de déchets de mangue et de déterminer la valeur alimentaire de ces produits pour animaux d'élevage, notamment des porcs.

Méthodologie et Résultats : Un volume de peau de mangue hachée d'une part et de pulpe+peau de mangue broyée d'autre part ont été mélangés respectivement à 1 et 1,25 volume de son, puis séchés au soleil (2-3 jours) et broyés pour obtenir des provendes de peau de mangue+son (PMS) et de pulpe+peau de mangue +son (PPS). Conservées pendant 8 mois dans des sacs elles ne se sont pas dégradées. La récupération de $2516 \mathrm{~kg}$ de déchets de mangues a permis la production de1850 kg d'aliments. La matière azotée totale du PMS $(10,61 \%)$ et du PPS $(8,72 \%)$ a été plus élevée que celle de la peau $(3,42 \%)$ et de la pulpe $(2,39 \%)$ et moins élevée que celle du son (12\%). l'Énergie Digestible (ED) du PMS (2826 kcal/kg MS) et du PPS (3204 kcal) ont été supérieures à celles de la peau (2280kcal) et inférieur à l'ED du son (2950 kcal) et de la pulpe (3802kcal/kg MS). Le son a été plus riche en Cellulose brute (35\%) que la pulpe, la peau, le PMS et le PPS. Conclusion et Applications : La récupération des déchets de mangues jadis jetés ouvre des perspectives de rendre plus disponible des aliments pour les animaux d'élevage, particulièrement les porcs. Elle permet d'améliorer la production de viande porcine et d'autres animaux à moindre coût. Cette récupération contribue à la lutte contre les mouches de fruit, à la réduction des risques de pollutions et à la création de valeur ajoutée pour la filière mangue. La mise au point d'une petite chaine mécanisée de production des aliments dans les vergers, dans les usines de transformation et sur les lieux de commercialisation permettra de récupérer de grandes quantités de déchets à moindre coût. Des travaux complémentaires à bonne période d'ensoleillement (avril, mai, juin) sont à entreprendre pour un meilleur rendement. 
Mots clés : Procédés de production, Déchets de mangues, Valeurs alimentaires, Aliments non conventionnels, Porcs, Burkina Faso.

Processes of production of nonconventional food for pig containing mangoes residues and determination of their food value in Burkina Faso

\begin{abstract}
Study objective: The aim of this study was to develop a production process of nonconventional livestock feed using mango residues, and to determine the nutritive value of the obtained feeds for livestock especially for pigs.

Methods and Results: One volume of shopped mangoes skin and one volume of mangoes pulp+skin, mixed with 1 and 1.25 volume of corn bran, respectively, were sun dried (2-3 days), and all ground to obtain the feed of skin+corn bran (PPS) and pulp+skin+corn bran (PPS). The obtained feeds were kept in bags during eight months and they were not degraded. The uses of $2516 \mathrm{~kg}$ of mangoes residues have allowed the production of $1850 \mathrm{~kg}$ of feeds. Crude protein of the PMS $(10.61 \%$,) and the PPS $(8.72 \%)$ were higher than those of mangoes skin $(3,42 \%)$ and of pulp $(2.19 \%)$ and lower than that of the corn bran (12\%). The digestible energy of PMS (2826 kcal $/ \mathrm{kg} \mathrm{MS}$ ) and of the PPS (3204 kcal $/ \mathrm{kg} \mathrm{MS}$ ) where higher than those of the skin (2281 kcal $/ \mathrm{kg}$ MS) and lower than those of the corn bran (2950 kcal/kg MS) and of the pulp (3802 kcal/kg MS). The corn bran was richer in cellulose (35\%) than pulp, skin, PMS and PPS.

Conclusion and applications: The use of mangoes residues (usually troughed) in the production of livestock feeds, open new perspectives for more animal feeds particularly for pigs. It will improve the production of low cost pig and other animals' meat. The recovery and use of mangoes residues will also contribute to strength against mango flies and therefore reduces pollution. This will also add a value to mango chain value. The development of small machine for the feeds production at the mango orchard, in the factories as well at the mangoes markets will allow the recovery and use of great quantities of mangoes residues. Future studies should be conducted during the hottest periods of the year (April, May and June) for better yields.

Keys Word: Processes of production, Nutritive value, Mangoes residues, Nonconventional feeds, Pigs, Burkina Faso.
\end{abstract}

\section{INTRODUCTION}

Dans les pays d'Afrique au Sud du Sahara (ASS), le déficit fourrager et nutritionnel de saison sèche, la mauvaise gestion des pâturages, le coût élevé et la faible disponibilité des sous-produits agricoles (SPA) et agro-industriels (SPAI), la faible valorisation des sous-produits agricoles et les mauvaises pratiques d'alimentation constituent des contraintes à la production animale (Mopaté et Koussou, 2003; MRA, 2007 ; Drabo, 2007 ; Kiendrébéogo, 2008 ; Mopaté et al., 2011). La mise à contribution de ces sous-produits, non consommés par l'homme, dans l'alimentation des animaux permettrait de mieux les valoriser (Preston, 1987) notamment chez les porcs qui sont reconnus être de bons transformateurs et qui valorisent mieux certains sous-produits de faible valeur (Bosma et al., 2004). Au Burkina Faso, l'alimentation est le principal facteur limitant du développement de l'élevage en général (MRA, 2007) et particulièrement celui de l'élevage porcin dans les zones urbaine et périurbaine (Kiendrébéogo et al., 2008). Dans la périphérie de Bobo-Dioulasso, ville située dans l'Ouest du Burkina Faso, les éleveurs de porcs font recours à une gamme variée d'aliments de bétail dont ceux non conventionnels comme la mangue (Kiendrébéogo, 2008). Ainsi, l'utilisation des sous-produits dans l'alimentation des animaux est une pratique courante, pour pallier certaines situations de carence alimentaire. Paradoxalement de grandes quantités de mangues pourrissent dans les vergers de l'Ouest du Burkina Faso, principale zone de production, à cause de la mouche de fruit qui engendre des pertes de l'ordre de 30 à 100\% (Ouédraogo, 2007). A ces pertes, s'ajoutent d'importantes quantités de mangues déclassées de la transformation à l'usine pour cause d'avaries et des déchets (peau, noyau et pulpe) issus de la de 
transformation représentant 20 à $40 \%$ des quantités transformées. En 2010 par exemple, sur 530 tonnes de mangue achetée par la coopérative agricole du Kénédougou (COOPAKE), environ 108 tonnes ont été avariées soit près de $30 \%$ de pertes. Cette perte sur la matière première qui est de 5,5 millions de $F$ $\mathrm{CFA}$, va créer un manque à gagner de 12 à 13 millions de F CFA sur le bilan de la campagne (lefaso.net, 2010). On constate cependant que les prélèvements directs par les animaux et les éleveurs en période d'abondance sont infimes, au regard des grandes quantités de mangues avariées et des déchets issus de la transformation. Or ces produits non conventionnels pourraient sous réserve de transformation, être utilisés dans l'alimentation des animaux. Pour une production nationale annuelle de 160314 tonnes de mangues, les pertes économiques (en amont et en aval), les problèmes de pollution et de gestion des déchets (en aval) sont importants. Le séchage au soleil de provendes obtenues par mélange de déchets de mangue (peau et pulpe) et de son (absorbant) permettrait de mieux valoriser ces déchets et les rendre plus disponibles

\section{MATÉRIEL ET MÉTHODES}

Site de l'étude et conditions environnementales d'expérimentation: L'étude a été réalisée dans une unité de séchage dénommée TENSYA/GUAMPRI basée à Toussiana, un département de la Province du Houet, dans la région des Hauts-bassins, situé à l'Ouest de Bobo-Dioulasso sur l'axe Bobo-Dioulasso-Banfora. Cette région bénéficie d'un climat de type soudanien. Elle constitue avec celle des Cascades, des grands bassins de production de mangues au Burkina Faso. L'agriculture pour l'alimentation des animaux d'élevage, en particulier les porcs. Cependant, la teneur élevée en eau de la mangue pouvant varier de $81,7 \%$ à $87,2 \%$ (Sawadogo-Lingani, 2001 ; Bally, 2006) induit des coûts élevés en énergie pour le séchage de l'ordre de 150-200FCFAlkg (Arnoldus et Floris, 2009). Le séchage au soleil minimiserait cette dépense énergétique à condition de recourir à un absorbant comme le son de céréale pour réduire la teneur en eau de la mangue et faciliter le séchage au soleil des provendes obtenues. Peu de travaux ont été consacrés à la mise au point de procédés de production de provendes à partir des déchets de mangues pour l'alimentation des animaux. Or la mise au point de tels procédés permettrait de créer de la valeur ajoutée à la filière mangue et de réduire la contrainte alimentaire par accroissement de la disponibilité des aliments pour les animaux d'élevage. Le présent article décrit la mise au point de procédés de production d'aliments non conventionnels (provendes) à base de déchets de mangues et d'en déterminer les valeurs alimentaires pour les animaux d'élevage, notamment les porcs.

est l'activité principale de la grande majorité de la population de cette région. Les spéculations agricoles concernent les céréales et les cultures de rentes (arachides et coton). La fabrication des provendes s'est déroulée au cours de la deuxième quinzaine du mois d'août 2011, période pendant laquelle quelques paramètres climatologiques ont été enregistrés (Tableau1)

Tableau1 : Conditions météorologiques descriptives de la zone de l'étude du 14 au 31 Août (Thomas, 2011)

\begin{tabular}{lcccc}
\hline Variables & \multicolumn{5}{c}{ Valeurs } \\
\hline & $\mathrm{N}$ & Minimum & Moyenne & Maximum \\
\cline { 2 - 5 } Température $\left({ }^{\circ} \mathrm{C}\right)$ & 18 & 24 & 25 & 28 \\
Humidité $(\%)$ & 18 & 71 & 84 & 92 \\
Vent $(\mathrm{m} / \mathrm{s})$ & 18 & 5 & 11 & 16 \\
Pluviométrie (mm) & 18 & - & 5 & 27 \\
Ensoleillement (heures/jour) & 14 & 1 & 5 & 9 \\
\hline
\end{tabular}

Démarche méthodologique de fabrication de
provendes

Récupération des déchets de mangue par absorption et séchage au soleil : Le taux d'humidité de la mangue est estimé à $81,7 \%$ (Bally, 2006) et parfois plus de 82 à 
$87,2 \%$ pour la variété Amélie (Sawadogo-Lingani, 2001). Pour éliminer cette grande quantité d'eau et permettre la conservation du produit sous forme sèche, cela implique une dépense élevée du point de vue énergie estimée de l'ordre de 150-200FCFA/kg de mangue séchée au Burkina Faso (Arnoldus et Floris, 2009). Le choix du séchage direct au soleil dans le cas de la présente recherche vise à minimiser la demande énergétique, partant à réduire les charges de récupération. En effet pour Talla et al. (2001) le séchage des fruits tropicaux en l'occurrence la banane et la mangue reste pour les pays africains en développement, un des moyens économiques de conservation de ces denrées pour lesquelles les pertes après récolte atteignent parfois $60 \%$. Compte tenu de la teneur élevée en eau de la mangue, du son de maïs vanné a été utilisé comme absorbant pour résorber une partie de l'eau en vue d'accélérer le séchage de la provende au soleil.

Procédés de fabrication des provendes : Deux types de provendes ont été fabriqués en tenant compte des ingrédients de mangue utilisés : peau de mangue ou peau de mangue + pulpe.

- Provende PMS (Peau de mangue + son) : la peau de mangue est un sous-produit provenant des unités de séchage de pulpe de mangue. Pour obtenir la pulpe de mangue à sécher, la peau est épluchée manuellement aux coutelets. Ce sous-produit disponible est ordinairement jeté en plein air. Ce sont ces épluchures qui ont été récupérées, découpées à la machette en petits morceaux puis mélangées à l'absorbant (son) à volume égal pour donner la provende PMS. Le PMS est ensuite bien malaxé dans des cuvettes puis étalé au soleil, sur des bâches en plastique, dans la journée jusqu'à 17 heures en vue d'obtenir un taux d'humidité n'excédant pas $14 \%$.

- Provende PPS (Pulpe et Peau de mangue+son): Des mangues avariées ont été d'abord broyées dans un broyeur conçu à cette fin, pour faciliter la séparation du noyau d'une part, de la pulpe et la peau de l'autre. Le broyeur permet de détacher le noyau sans l'éclater pour éviter le mélange de l'amende du noyau avec le broyat (pulpe+ peau). Une fois détachés, ces noyaux sont retirés du broyat. Par la suite, 01 volume du broyat de pulpe +peau a été mélangé à 1,25 volume de son. La provende PPS ainsi obtenue est bien malaxée dans des cuvettes puis séchée au soleil sur des bâches en plastique dans la journée jusqu'à 17 heures en vue d'obtenir un taux d'humidité de moins de $14 \%$. Les itinéraires techniques de préparation des provendes PMS (peau de mangue+son) et PPS (pulpe+peau de mangue+son) sont représentés dans les Planches 1 et 2 ci-dessous : 


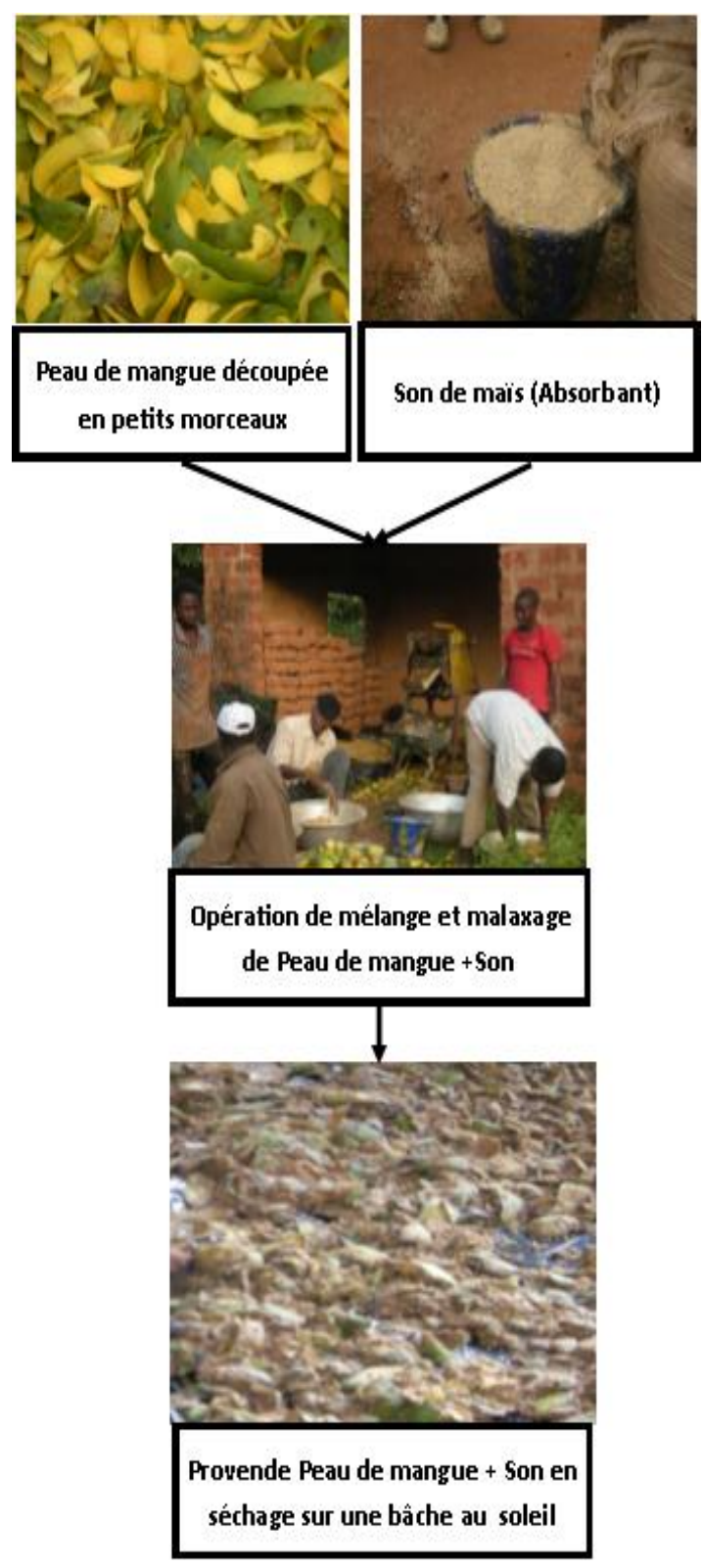

Planche 1 : Procédé de préparation de la provende PMS (peau de mangue + son)

Les PMS et PPS ainsi obtenus ont été broyés à l'aide d'un moulin à mil pour obtenir de la farine de $4 \mathrm{~mm}$ de diamètre.

\section{- Méthodologie de conservation des provendes}

Pour assurer une meilleure conservation des aliments, les provendes ont été conditionnées par la méthode du triple ensachage : un premier sac contient le produit, un

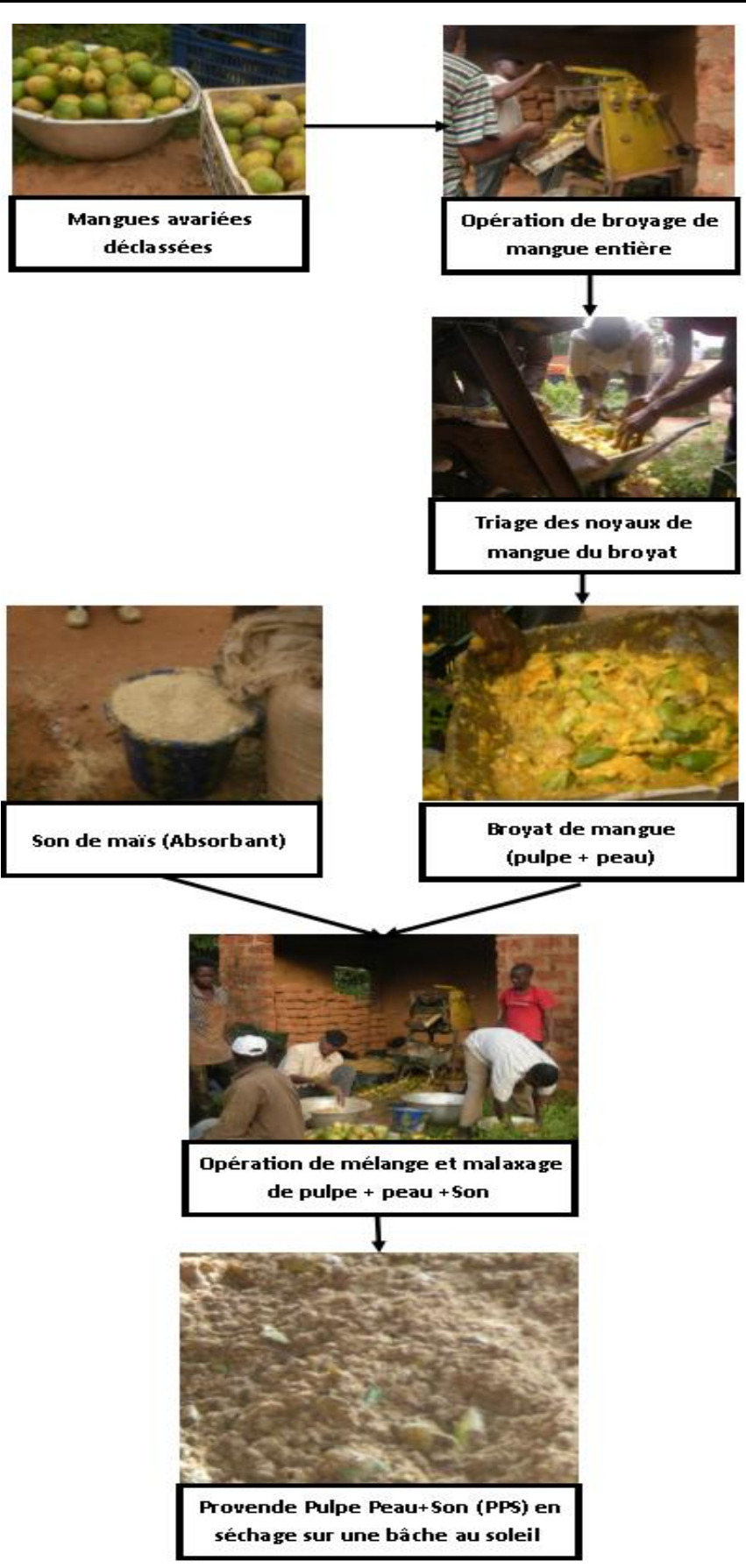

Planche 2 : Procédé de préparation de la provende PPS (pulpe+peau de mangue+son)

second dans lequel on met le premier qui contient le produit et le tout placé dans un troisième sac en plastique tissé. Cette technologie empêche toute entrée d'air, d'où la mort des insectes s'y trouvant et l'arrêt de leur cycle de reproduction (RECA, 2012). Les provendes ont ensuite été conservées pendant 08 mois (septembre 2011 à avril 
2012) avant leur utilisation dans un essai de rationnement à des porcs en croissance.

Composition chimique et calcul des teneurs en Énergie des aliments : La composition chimique (MS, MAT, CB, Ca, P, InsCh, ADF, NDF, NaCl) des ingrédients de base (son, peau de mangue et pulpe), des provendes (PMS et PPS) a été obtenue par analyse d'échantillons suivant les méthodes de AOAC (1975) décrit par Bougoum (2000) et Kaboré-Zoungrana et al. (2008). La matière sèche (MS) a été obtenue après séchage à l'étuve à $105^{\circ} \mathrm{C}$ pendant $24 \mathrm{~h}$ et les cendres totales (MM) par calcination de la matière sèche à $550^{\circ} \mathrm{C}$. Les matières azotées totales (MAT) correspondent à l'azote selon Kjeldahl $(\mathrm{N} \times 6,25)$; la cellulose brute selon Weende et les constituants pariétaux, NDF (neutral détergent fiber), ADF (acid detergent fiber) dosé directement sur l'échantillon et ADL (acid detergent lignin) sulfurique déterminé à partir de l'ADF. Par ailleurs des éléments minéraux tels que le phosphore $(P)$ et le calcium $(\mathrm{Ca})$ ont été dosés. L'utilisation prochaine des

\section{RÉSULTATS}

Production de provendes : coûts de production et récupération des déchets de mangue : La production des provendes (quantités et coûts) est présentée dans le Tableau 2. L'opération a permis de produire $959 \mathrm{~kg}$ de PMS à partir de $1183 \mathrm{~kg}$ de peau de mangue récupérée et $891 \mathrm{~kg}$ de PPS à partir de $1333 \mathrm{~kg}$ de peau + pulpe. La provendes dans un essai de rationnement de porcs pour améliorer la production de viande a conduit à l'évaluation de leur valeur énergétique théorique. L'Energie brute (EB) et l'Energie digestible (ED) ont été obtenues par calcul, par application des équations de prédiction données par Henry (1971) pour l'EB et ED. Les calculs ont utilisés les résultats d'analyse des échantillons (Tableau 3) :

$$
\begin{array}{ll}
E B=5,74 M A T+9,50 M G+4,79 C B+4,17 E N A \pm 0,9 \% \text { avec } \\
- & E B=\text { Energie brute } \\
- & M A T=\text { Matière azotée totale } \\
- & M G=\text { Matière grasse } \\
- & \mathrm{CB}=\text { Cellulose brute } \\
- & \mathrm{ENA}=\text { Extractif non azoté } \\
\text { ED }\left(\frac{\text { koal }}{\mathrm{KgMS}}\right)=\frac{\text { EBxdE }}{100} \text { avec } \\
\text { - } \\
\mathrm{dE}=\text { digestibilité de l'énergie avec } \mathrm{dE}=96,36- \\
0,872 \mathrm{NDF}(\mathrm{R}=-0,947)
\end{array}
$$

production totale (PMS+PPS) de $1850 \mathrm{~kg}$ de provende a nécessité 1405 tonnes de son de maïs. Le coût de production de la provende PMS a été de 100,9 F CFA/kg. Quant à la provende PPS, elle a été produite à 147,3 F CFA/kg toutes charges comptabilisées.

Tableau 2 : Coûts (FCFA) du kg de provendes produites à partir des déchets de mangue récupérés

\begin{tabular}{lcc}
\hline Ingrédients & \multicolumn{2}{c}{ Aliments } \\
\cline { 2 - 3 } & PPS (FCFA) & PMS (FCFA) \\
\hline Peau +pulpe frais & 25 & - \\
Peau de mangue fraîche & - & 6,25 \\
Son de maïs & 51,67 & 51,67 \\
Main d'œuvre & 66,38 & 41,11 \\
Amortissement du matériel et équipement & 4,2 & 1,89 \\
Montant & $\mathbf{1 4 7 , 2 5}$ & $\mathbf{1 0 0 , 9 3}$ \\
\hline
\end{tabular}

Valeurs alimentaires des ingrédients et des aliments incorporant des déchets de mangue : La composition chimique des ingrédients et provendes PMS et PPS (Tableau 3) ont montré que les teneurs en eau des provendes PMS et PPS sont faibles et inférieur à 10\% permettant leur stockage et conservation. L'adjonction du son de maïs (ingrédient le plus riche en MAT) a permis d'améliorer les teneurs en MAT des provendes qui sont de $10,61 \%$ et $8,72 \%$ respectivement pour le PMS et le PPS. Cette adjonction a par contre conduit à des teneurs en CB plus importantes. Les teneurs en MG ont été aussi considérablement augmentées et sont de $7,65 \%$ et $5,42 \%$ respectivement pour le PMS et PPS. Les teneurs en EB calculées des provendes sont élevées avec celle du PMS (4457kcal/kgMS) supérieure à celle du PPS (4374kcal/kgMS). Cependant l'examen des teneurs en ED fait apparaitre une inversion. En effet la valeur de $\mathrm{dE}$ du PPS (73\%) est supérieure de 10 point à celle du PMS (63\%). 
Tableau 3 : Composition chimique (\% MS) des ingrédients et des provendes (PMS et PPS)

\begin{tabular}{lccrcc}
\hline Teneurs & Pulpe & Peau de mangue & Son & PMS & PPS \\
\hline Matières sèches & 93,62 & 78,68 & 96 & 90,78 & 91,77 \\
Matières azotées totales & 2,19 & 3,42 & 12 & 10,61 & 8,72 \\
Matières grasses & 0,46 & 0,37 & 12 & 7,65 & 5,42 \\
Cellulose brute & 2,66 & 8,33 & 35 & 9,35 & 8,59 \\
NDF & 6,17 & 44,77 & 43 & 37,57 & 26,46 \\
ADF & 3,82 & 17,08 & 40 & 15,01 & 14,35 \\
Lignine & 2,13 & 6,54 & 4 & 4,78 & 3,34 \\
Matières minérales & 1,59 & 7,11 & 5 & 8,25 & 6,56 \\
Insoluble chlorhydrique & 0,16 & 4,89 & 1 & 4,54 & 2,2 \\
Calcium & 0,21 & 0,39 & 1 & 0,43 & 0,44 \\
Phosphore & 0,28 & 0,33 & 1 & 0,18 & 0,15 \\
NaCl & 0,64 & 1,17 & 1 & 1,23 & 0,43 \\
EB (kcal/kgMS) & 4179 & 3998 & 5000 & 4457 & 4374 \\
ED (kcal/kgMS) & 3802 & 2281 & 2951 & 2826 & 3204 \\
\hline
\end{tabular}

NDF= Neutral Digestible Fiber ; ADF= AcidDigestible Fiber ; ED=Énergie Digestible ; PMS= Provende de peau de mangue ; MES= Provende de mangue entière

\section{DISCUSSION}

Nos résultats démontrent la récupération possible d'importantes quantités de déchets de mangue (peau) et de mangues avariées qui sont écartées de la transformation et qui pourraient servir à la fabrication de provendes en leur adjoignant du son de maïs comme absorbant. L'adjonction de l'absorbant permet d'augmenter les quantités de matière sèche et de réduire en conséquence la durée de séchage à 27 heures contre $63 \mathrm{~h}$ pour le séchage au soleil de la peau. Les provendes séchées au soleil ont des teneurs en eau assez proches de celle des mangues séchées au four $(6,39 \%)$ et corroborent les prévisions de Talla et al. (2001) qui indiquent que la banane et la mangue peuvent être séchés jusqu'à un niveau de teneur en eau inférieur à $20 \%$. Les provendes produites ont des teneurs en MS élevées et adéquates pour permettre leur stockage et leur conservation. Leurs teneurs sont inférieures au $14 \%$ d'humidité au-delà desquelles la stabilité d'un produit à la température ambiante n'est pas assurée (Arnoldus et Floris, 2009).

Le recours à la source énergétique solaire gratuite a aussi permis une économie en dépense énergétique par rapport au gaz, habituellement utilisé pour le séchage des mangues. De plus, cette efficacité pourrait être améliorée si les traitements débutaient à partir du mois d'avril, période de démarrage de la campagne de récolte et de transformation des mangues au Burkina Faso. En effet la période des mois d'avril et mai est plus ensoleillée et sèche, avec une pluviométrie très faible comparativement à celle de la période de nos travaux (août). En matière de valeurs nutritionnelles des aliments préparés, les provendes PMS et PPS sont d'une teneur respective plus importante en ED que l'ingrédient peau et les ingrédients peau et son. Ainsi, les provendes obtenues ont amélioré les teneurs en énergie des ingrédients, permettant de couvrir les besoins énergétiques du porc. L'apport d'énergie alimentaire représente le principal coût de production (plus de 50\%) chez le porc (Noblet et al., 2003). En effet, les teneurs en ED des provendes PMS $(2826 \mathrm{kcal} / \mathrm{kg})$ et PPS (3204 Kcal $/ \mathrm{kg})$ sont supérieures à l'offre énergétique moyenne de rations $(2518 \mathrm{kcal} / \mathrm{kg})$ distribuées aux porcs dans les élevages intensifiés de la périphérie de Bobo-Dioulasso (Kiendrébéogo, 2006). La teneur en ED des deux provendes est inférieure mais proche de l'ED du maïs (une des principales sources énergétique pour l'alimentation des monogastriques) disponible pour des porcs en croissance (3390kcal) et la truie (3530kcal) (INRA, 2004). En dehors du son, cette tendance est également observée pour la MAT des provendes comparées à leurs ingrédients. En revanche, la provende PMS contient 7 fois plus de MAT que l'ingrédient peau et plus d'une fois moins que le son. Les teneurs en MAT des provendes sont plus élevées que celle de la peau $(6,28 \%)$ rapportée par Omojowo (2010). 
La faible teneur en CB des provendes PMS et PPS comparée à la teneur en $\mathrm{CB}$ des principaux aliments des porcs au Burkina Faso comme le son (35\%) et la drêche de bière locale $(19 \%)$ et leurs $\mathrm{dE}$ plus élevées que ces derniers ( $42 \%$ pour la drêche et $59 \%$ pour le son) (Communication personnelle) préfigure d'une meilleure consommation volontaire (CV) et valorisation par les porcs. Leur incorporation dans des rations d'engraissement peut favoriser la production de viande maigre, recherchée par les producteurs africains et asiatiques pour répondre à la demande des consommateurs (Wuntu, 1993 ; Centres, 1996 ; Patricot, 2002 et Kiendrébéogo et al., 2012). Les coûts de production des aliments PPS (147,25 F CA/kg) et PMS $(100,93 \mathrm{~F} \mathrm{CFA} / \mathrm{kg})$ ont été inférieurs au seul coût de l'énergie (150-200 F CFA) nécessaire pour sécher un $\mathrm{kg}$ de mangue au four (Arnoldus et Floris, 2009). Ces coûts sont également inférieurs aux prix de vente du maïs (150-

\section{CONCLUSION}

L'étude a permis la mise au point de procédés de valorisation des déchets de mangue par la fabrication de provendes et d'en déterminer leurs valeurs alimentaires pour les animaux d'élevage, notamment les porcs. La valorisation des déchets peu ou pas utilisés permet de rendre plus disponible des aliments de valeurs alimentaires intéressantes pour les animaux d'élevage, particulièrement pour l'élevage porcin en toute saison. Elle contribuera à la lutte contre la mouche de fruit, à réduire les risques de pollution et à créer de la valeur ajoutée pour la filière mangue. L'incorporation des provendes dans des rations destinées aux animaux, notamment aux porcs, permettra d'améliorer la production de viande à moindre coût et d'engranger un plus valu aux producteurs. II s'agit là d'une première et importante phase qui mérite d'être affinée pour renforcer l'efficacité du séchage et réduire les coûts de production.

\section{REMERCIEMENTS}

Nous remercions tous ceux qui ont contribué à la réalisation de ce travail. En particulier :

Madame Coulibaly de l'usine de séchage de mangue TENSYA/GUAMPRI de Toussiana pour sa contribution matérielle et financière à la réalisation du projet ;

\section{BIBLIOGRAPHIE}

Arnoldus M, Floris VP, 2009. L'amélioration de la performance des produits transformés de la mangue au Burkina Faso et au Mali. Rapport d'étude.116p.
200FCFA $/ \mathrm{kg}$ ), du poisson (300 à $350 \mathrm{~F} \mathrm{CFA} / \mathrm{kg}$ ), de la graine de soja (250 FCFA $/ \mathrm{kg}$ ) qui sont les principaux aliments localement utilisés dans l'alimentation des porcs et les autres animaux (FAO, 2012). Les coûts de production du PMS et du PPS sont cependant inférieurs aux prix d'achat du son de maïs, à Bobo-Dioulasso, de 18 à $25 \mathrm{FCFA} / \mathrm{kg}$ (à toute saison sèche) rapporté par Bougoum en 2000. En 2011 les prix du son rapportés par Sanogo et Sanou étaient d'en moyenne 23 FCFA $/ \mathrm{kg}$ chez les meuniers et de 60 à 75 FCFA $/ \mathrm{kg}$ chez les revendeurs. Les aliments PMS et PPS sont également plus chers que les drêches de dolo sèches qui coûtent en moyenne 17 FCFA/kg à Bobo-Dioulasso (Sanou et Sanogo, 2011). En somme et au regard des valeurs alimentaires des provendes, l'adjonction du son de maïs aux déchets de mangues permet l'obtention d'aliments aux teneurs énergétiques et azotées plus importantes que celles de la moyenne des ingrédients utilisés dans leur production.

La mise au point d'une petite chaine mécanisée et automatisée de production, autonome dans les vergers, dans les usines de transformation et les lieux de commercialisation permettra la récupération de grandes quantités de déchets. Ces recherches supplémentaires permettront en outre d'analyser la stabilité des caractéristiques physico-chimiques et hygiéniques (microbiologique en particulier) des provendes. Le séchage au soleil a permis d'avoir des provendes aux taux d'humidité voisins de celui de la pulpe séchée au soleil pendant une semaine mais surtout, d'économiser en dépense énergétique s'ils étaient séchés au gaz. Différents taux d'incorporation du son et différents types d'absorbants pourront être testés en vue d'identifier les meilleurs traitements. Ceux-ci pourront permettre de récupérer de grandes quantités de déchets de mangue à moindre coût.

Dr El Adj Traoré, Dr Sali NGom, madame Adjara Diarra du Laboratoire National de l'Elevage et de Recherches Vétérinaires (LNERV) de l'Institut Sénégalais de Recherches Agricoles (ISRA) à Dakar (Sénégal) et monsieur Léonard Savadogo pour leur contribution à l'analyse des échantillons.

http://www.euacpcommodities.eu/files/1WSTD05Rapport \%20fili\%C3\%A8re\%20mangue $\% 20$ transform $\%$ C3\%A9e\%20FRENCH.pdf (29/12/2012).RoyalTropical institute. 
Bally, 2006.Species Profiles for Pacific Island Agroforestry: Mangifera indica (mango) Anacardiaceae (cashew family).http://www.traditonaltree.org(12/12/2012)

Bosma RH, Zongo LC, Sané A, Zoungrana C, Soudré A, 2004. Comparaison participatoire de trois méthodes d'engraissement des porcs dans les provinces du Sanguié et du Boulkiemdé au Burkina Faso.LivestockResearch for Rural Development, Vol. 16, №2, Février 2004. http://www.Irrd.org//rrd16/2/cont1602.htm

Bougoum A, 2000. Contribution des issues de céréales et des fourrages dans l'alimentation des animaux des élevages périurbains. Mémoire d'Ingénieur d'Elevage, UPB/IDR, BoboDioulasso, Burkina Faso. http://www.greenstone.refer.bf/collect/upb/index/ assoc/HASH01d7.dir/IDR-2000-BOUCON.pdf(23/05/2013)

Centres J M, 1996. L'élevage et l'agriculture en zones urbaines et périurbaines dans deux villes sahéliennes: Bamako et Bobo-Dioulasso. Cahiers Agricultures, 5 (5) : $373-381$

Drabo, 2007. Contraintes actuelles et perspectives de l'élevage porcin dans les zones intra et périurbains de Ouagadougou au Burkina Faso. Mémoire d'Ingénieur Zootechnicien. Institut polytechnique Rural de Formation et de Recherche Appliquée (IPR//FRA) de Katibougou, Annexe de Bamako, Université du Mali, Bamako. 68p

FAO. 2012. Secteur Porcin Burkina Faso. Revues nationales de l'élevage de la division de la production et de la santé animales de la FAO. No. $1 . \quad$ Rome. http://www.fao.org/docrep/015/i2567f/i2567f00.p df $(23 / 05 / 2013)$

Henry $Y, 1971$. Essai de prévision de la valeur en énergie digestible des aliments pour le porc à partir de leur teneur en constituants membranaires.http://journees-rechercheporcine.com/texte/1971/71txtAlim/A7102.pdf (18/06/2013)

Kaboré-Zoungrana C, Diarra B, Adandedjan C, Savadogo S, 2008 : Valeur nutritive de Balanites aegyptiaca pour l'alimentation des ruminants. Livestock Research for Rural Development. Volume 20, Article \#56. Retrieved June 19, 2013,

from
http://www.Irrd.org//rrd20/4/kabo20056.htm $(18 / 06 / 2013)$

Kiendrébéogo T, 2006. Diagnostic des élevages porcins de la zone de Bobo-Dioulasso: systèmes d'élevage et conditions technico-économiques de production. Mémoire de DEA, Université polytechnique de Bobo-Dioulasso. 48p.

Kiendrébéogo T, Hamadou S, Mopaté LY, KaboréZoungrana C-Y, 2008. Typologie des élevages porcins urbains et périurbains de BoboDioulasso (Burkina Faso). Revue Africaine de Santé et des Productions Animales (RASPA), 6 (3-4) : $205-212$

Kiendrébeogo T, Mopaté Logténé $\mathrm{Y}$, Kondombo S R, Kaboré-Zoungrana C-Y, 2012b. Approvisionnement en porcs vifs et viande porcine de la ville de Bobo-Dioulasso (Burkina Faso). Journal of Agriculture and Environment for International Development (JAEID), 106 (2) : 105-122

lefaso.net, 2010. Production de mangues au Burkina Faso : la mouche de fruit fait des dégâts. Article in lefasonet en date du 3/08/2010. http://www.lefaso.net/spip.php?article37905 (11/12/2012).

Mopaté LY, Koussou MO, 2003. L'élevage porcin, un élevage ignoré mais pourtant bien implanté dans les agro-systèmes ruraux et périurbains du Tchad. In : Jamin J. Y., Seyni Boukar L. et Floret C. (éditeurs scientifiques -CD-ROM), Actes du colloque « Savanes africaines : des espaces en mutations, des acteurs face à des nouveaux défis », Garoua, Cameroun, 27 -31/05/2002, 9 $p$.

Mopaté LY, Kaboré-Zoungrana C-Y, Facho B, 2011. Disponibilité et valeurs alimentaires des sons de riz,maïs et sorgho mobilisables dans l'alimentation desporcs à N'Djaména (Tchad). Journal of Applied Biosiences 41: 2757 2764p.

Mopaté LY, Kaboré-Zoungrana C-Y, Facho B, 2011. Disponibilité et valeurs alimentaires des drêches artisanales et résidus d'alcool traditionnel mobilisables dans l'alimentation desporcs à N'Djaména (Tchad). Journal of Applied Biosiences 42: 2859-2866.

MRA, 2007. Diagnostic des filières bétail-viande et petits ruminants du Burkina Faso.Rapport final, AGRER-Statistika, $145 \mathrm{p}$. 
Noblet J, Bontems V, Tran G, 2003. Estimation de la valeur énergétique des aliments pour le porc. In Production Animale 16(3), 197-2010.

Omojowo TM, Omojowo FS and Alatise PS, 2010. Growth response and nutritional evaluation of mango peel-based diets on Tilapia (Oreochromis Niloticus) Fingerlings. Article in Researcher 2012, 2(6) 44- 49.

http://www.sciencepub.net/researcher/research0206/06 3047research0206_44_49.pdf (4/12/2012)

Ouédraogo SN, 2007. Étude des attaques des mouches de fruits (Diptera tephritidae) sur la mangue dans la province du Kénédougou (Ouest du Burkina Faso). Mémoire de diplôme d'étude approfondie(DEA) en gestion intégrée des Ressources naturelles(GIRN) à l'Institut du développement Rural (IDR) de l'Université Polytechnique de Bobo-Dioulasso(UPB), 2007, $70 \mathrm{p}$.

Patricot S, 2002. Les systèmes d'élevages familiaux de porcs au Nord-Viêtnam. Synthèse bibliographique. DESS Productions animales en régions chaudes. Année Universitaire, 2001 2002, Cirad-Emvt, Montpellier, France, 38 p. + annexes $21 \mathrm{p}$.

Preston TR, 1987. Porcs et volailles sous les tropiques: utilisation des ressources alimentaires locales. Centre technique de coopération agricole et rurale (CTA), Wageningen, Pays-Bas, $27 \mathrm{p}$.

RECA (Réseau National des Chambres d'Agriculture du Niger), 2012. Le triple ensachage pour la conservation du niébé - Un point de situation. Note d'information / Technologies et techniques $n^{\circ} 2.7 p$.

Sauvant D, Perez J.-M, Tran G, 2004. Table de composition et de valeur nutritive des matières premières destinées aux animaux d'élevage, porcs, volailles, bovins, ovins, caprins, lapins, chevaux, poissons. Ouvrage, $2^{\mathrm{ème} e}$ édition revue et corrigée, INRA, Paris, 2004. 301p.

Sanogo D, Sanou B, 2011. Disponibilité des aliments mobilisables dans l'alimentation des porcs à Bobo-Dioulasso (Burkina Faso). Rapport de stage. Institut de Développement Rural (IDR) de l'Université Polytechnique de Bobo-Dioulasso (UPB). 32p.

Talla A, Jannot Y, Kapseu C, Nganhou J, 2001. Étude expérimentale et modélisation de la cinétique de séchage de fruits tropicaux: Application à la banane et à la mangue. Article in revue Sciences des Aliments, 21(2001) 499-518p.
Thomas, 2011. Observations, Prévisions, modèles en temps réel. Données mensuelles de août 2011 pourBobo-Dioulasso( Burkina Faso). http://www.meteociel.be/climatologie/villes.php? code $=65510 \&$ mois $=8 \&$ annee $=2011$ Consulté le 29/12/ 2012, sur Meteociel.fr

Wuntu Norrytha Lineke, 1993. Etude du rôle des fibres dans l'alimentation porcine. Synthèse bibliographique. DESS Productions animales en régions chaudes. Année universitaire 19921993, Cirad-Emvt, Maisons-Alfort, France, 12 p. 